PROCEEDINGS OF THE

AMERICAN MATHEMATICAL SOCIETY

Volume 135, Number 1, January 2007, Pages 211-216

S 0002-9939(06)08456-5

Article electronically published on June 29, 2006

\title{
ON AFFINE FRAMES WITH TRANSCENDENTAL DILATIONS
}

\author{
R. S. LAUGESEN
}

(Communicated by Joseph A. Ball)

\begin{abstract}
We answer a question of $O$. Christensen about affine systems in $L^{2}(\mathbb{R})$. Specifically, we show that if the dilation factor $a>1$ is transcendental, then cancellations cannot occur between different scales, in the conditions for the affine system to form a frame. Such cancellations are known to occur when $a$ is an integer.
\end{abstract}

\section{INTRODUCTION}

Consider a generating function $\psi \in L^{2}=L^{2}(\mathbb{R})$ and a dilation factor $a>1$. Define

$$
\psi_{j, y}(x)=a^{j / 2} \psi\left(a^{j} x-y\right), \quad j \in \mathbb{Z}, \quad y \in \mathbb{R}, \quad x \in \mathbb{R} .
$$

Call $\left\{\psi_{j, k}: j, k \in \mathbb{Z}\right\}$ the affine system generated by $\psi$ and $a$.

We are interested in when the affine system forms a frame for $L^{2}$, in other words, when $\psi$ is a frame wavelet. Recall that a countable collection of functions $\left\{g_{m}\right\}$ in $L^{2}$ forms a frame if constants $0<A \leq B<\infty$ exist such that

$$
A\|f\|^{2} \leq \sum_{m}\left|\left\langle f, g_{m}\right\rangle\right|^{2} \leq B\|f\|^{2} \quad \text { for all } f \in L^{2},
$$

where $\|f\|$ denotes the $L^{2}$-norm of $f$. The numbers $A$ and $B$ are frame bounds. The frame is tight if $A=B$. For example an orthonormal basis forms a tight frame with $A=B=1$.

The affine system $\left\{\psi_{j, k}\right\}$ forms a frame if and only if $0<A_{\psi} \leq B_{\psi}<\infty$, where

$$
A_{\psi}:=\inf _{\|f\|=1} \sum_{j, k \in \mathbb{Z}}\left|\left\langle f, \psi_{j, k}\right\rangle\right|^{2}, \quad B_{\psi}:=\sup _{\|f\|=1} \sum_{j, k \in \mathbb{Z}}\left|\left\langle f, \psi_{j, k}\right\rangle\right|^{2} .
$$

In his survey on frame theory, O. Christensen [8] inquired whether formulas for $A_{\psi}$ and $B_{\psi}$ could be found that involve "cancellation" between terms at different dilation scales (different values of $j$ ). Such cancellation is known to occur when $a$ is an integer. This paper shows it cannot occur when the dilation $a$ is transcendental.

Quick tutorial on frames. Frame theory began with Duffin and Schaeffer [12], who were motivated by nonharmonic Fourier series. Frames have become particularly important in the last 20 years in connection with Gabor systems (time-frequency

Received by the editors June 30, 2005 and, in revised form, July 31, 2005.

2000 Mathematics Subject Classification. Primary 42C40; Secondary 42C15.

Key words and phrases. Affine system, frame, transcendental.

This work was completed during a Visiting Erskine Fellowship at the University of Canterbury, New Zealand, and also with support from National Science Foundation award DMS-0140481.

(C)2006 American Mathematical Society Reverts to public domain 28 years from publication 
analysis) and affine systems (wavelet analysis). See for example the books [13, 16], the survey articles [5, 8], and the open problems in Section [5 below.

Note that if the $\left\{g_{m}\right\}$ form a frame, then every $f \in L^{2}$ has a reconstruction $f=\sum_{m} c_{m} g_{m}$ as a linear combination of the $g_{m}$. A frame need not be a basis, though: these coefficients $c_{m}$ need not be unique. One choice of coefficients is $c_{m}=\left\langle f, h_{m}\right\rangle$, where the $\left\{h_{m}\right\}$ form a "dual frame".

Frames are at first sight less elegant than orthonormal bases, due to their redundancy or overcompleteness, but this redundancy gives them considerable power in applications such as noise reduction [2].

Frame theory in finite-dimensional spaces has flowered recently too. See [3, 6, and the results on signal reconstruction without phase information in [1].

\section{Results}

Recall that a real number is algebraic if it is a root of some nontrivial polynomial equation with integer coefficients. Otherwise the number is transcendental. The algebraic numbers are countable, and thus most real numbers are transcendental.

Define a dense subclass of the unit sphere in $L^{2}$ by

$\mathcal{C}=\left\{f \in L^{2}:\|f\|=1\right.$, and $\hat{f}$ is bounded and compactly supported in $\left.\mathbb{R} \backslash\{0\}\right\}$, and for $f \in L^{2}$ define a family of nonnegative 1-periodic functions by

$$
\left(T_{j} f\right)(z)=\sum_{k \in \mathbb{Z}}\left|\left\langle f, \psi_{j, k-z}\right\rangle\right|^{2}, \quad j \in \mathbb{Z}, \quad z \in \mathbb{R} .
$$

$T_{j} f$ is continuous if $f \in \mathcal{C}$, by [17, Lemma A.4].

Theorem 2.1. Assume $\psi \in L^{2}$ and $a>1$. Then

$$
B_{\psi} \leq \sup _{f \in \mathcal{C}} \sum_{j \in \mathbb{Z}} \max T_{j} f
$$

with equality if $a$ is transcendental. Also, if $B_{\psi}<\infty$, then

$$
A_{\psi} \geq \inf _{f \in \mathcal{C}} \sum_{j \in \mathbb{Z}} \min T_{j} f
$$

with equality if a is transcendental.

Theorem 2.1 implies a sufficient frame condition involving only $\psi$, and not $f$.

Corollary 2.2 (Casazza and Christensen [7, Theorem 2.5], 8, Theorem 5.1]). Assume $\psi \in L^{2}$ and $a>1$. Then

$$
B_{\psi} \leq B:=\text { ess. } \sup _{1 \leq|\xi| \leq a} \sum_{j \in \mathbb{Z}}\left(\left|\widehat{\psi}\left(\xi a^{j}\right)\right|^{2}+\sum_{n \neq 0}\left|\widehat{\psi}\left(\xi a^{j}\right) \widehat{\psi}\left(\xi a^{j}-n\right)\right|\right) .
$$

Also if $B_{\psi}<\infty$, then

$$
A_{\psi} \geq A:=\text { ess. } \inf _{1 \leq|\xi| \leq a} \sum_{j \in \mathbb{Z}}\left(\left|\widehat{\psi}\left(\xi a^{j}\right)\right|^{2}-\sum_{n \neq 0}\left|\widehat{\psi}\left(\xi a^{j}\right) \widehat{\psi}\left(\xi a^{j}-n\right)\right|\right),
$$

provided the sum over $j$ in this quantity $A$ is well defined. 
Corollary 2.2 implies that if $0<A \leq B<\infty$, then $\psi$ generates an affine frame.

When $a=2$, the bounds in Corollary 2.2 can be replaced by ones in which summations over certain $j$ and $n$ values are carried out inside the absolute value signs. This is due to Ph. Tchamitchian, as presented by I. Daubechies in [11, Theorem 2.9]. Getting these summations inside the absolute value signs can lead to helpful cancellations [11, p. 984].

Christensen [8, p. 288] wrote that it "would be interesting if [Corollary 2.2] could be generalized that way" for general $a>1$. Theorem 2.1 makes such a generalization impossible when $a$ is transcendental, because the max and min are inside the sum over $j$ and so oscillations in different $T_{j} f$ functions cannot possibly cancel each other. (This statement is best understood in terms of formula (3.1) in the proof below.)

Theorem 2.1 leaves open the possibility of modifying Corollary 2.2 to allow cancellations within the sum over $n$. But it is unclear how to do that.

Notes.

1. It is an open question whether equality must hold in Theorem 2.1 whenever $a^{j} \notin \mathbb{Q}$ for all $j>0$. This condition is strictly weaker than transcendentality of $a$. For example $a=1+\sqrt{2}$ is algebraic rather than transcendental, being a root of $a^{2}=2 a+1$, but $a^{j} \notin \mathbb{Q}$ for all $j>0$. Incidentally, the condition $a^{j} \notin \mathbb{Q}$ for all $j>0$ already arises in tight frame characterizations [9, §3].

2. If $a^{j} \in \mathbb{Q}$ for some $j>0$, then cancellations can occur in the style of Tchamitchian's result, basically because the Fourier series of $T_{j} f\left(a^{j} z\right)$ has terms such as $\exp \left(2 \pi i n a^{j} z\right)$, and it is possible to have $n_{1} a^{j_{1}}=n_{2} a^{j_{2}}$ (when $a^{j_{1}-j_{2}}=$ $\left.n_{2} / n_{1} \in \mathbb{Q}\right)$.

\section{Proof of Theorem 2.1}

Note that $B_{\psi}=\sup _{f \in \mathcal{C}} \sum_{j, k \in \mathbb{Z}}\left|\left\langle f, \psi_{j, k}\right\rangle\right|^{2}$, by definition (1.1) of $B_{\psi}$ and the density of $\mathcal{C}$ in the unit sphere of $L^{2}$, using [17, Lemma B.1]. Hence

$$
B_{\psi}=\sup _{f \in \mathcal{C}} \sum_{j \in \mathbb{Z}} T_{j} f(0) \leq \sup _{f \in \mathcal{C}} \sum_{j \in \mathbb{Z}} \max T_{j} f .
$$

For the equality statement in the theorem, we start with two trivial but important observations: if $f \in \mathcal{C}$, then the translates $f(\cdot-z)$ of $f$ also belong to $\mathcal{C}$ whenever $z \in \mathbb{R}$, and $\left\langle f(\cdot-z), \psi_{j, k}\right\rangle=\left\langle f, \psi_{j, k-a^{j} z}\right\rangle$. Therefore

$$
\begin{aligned}
B_{\psi} & =\sup _{f \in \mathcal{C}} \sup _{z \in \mathbb{R}} \sum_{j \in \mathbb{Z}} T_{j}(f(\cdot-z))(0) \\
& =\sup _{f \in \mathcal{C}} \sup _{z \in \mathbb{R}} \sum_{j \in \mathbb{Z}} T_{j} f\left(a^{j} z\right) .
\end{aligned}
$$

The equality statement for $B_{\psi}$ in Theorem 2.1 now follows from the next lemma.

Lemma 3.1. If $a \in \mathbb{R}$ is transcendental and the functions $h_{j}: \mathbb{R} \rightarrow \mathbb{R}$ are continuous, nonnegative and 1-periodic, then

$$
\sup _{z \in \mathbb{R}} \sum_{j \in \mathbb{Z}} h_{j}\left(a^{j} z\right)=\sum_{j \in \mathbb{Z}} \max h_{j} .
$$


Proof of Lemma 3.1, The " $\leq$ " direction is immediate. For the " $\geq$ " direction, the task is to show that for each $J \in \mathbb{N}$

$$
\sup _{z \in \mathbb{R}} \sum_{|j| \leq J} h_{j}\left(a^{j} z\right)=\sum_{|j| \leq J} \max h_{j} .
$$

To accomplish this, write $m_{j} \in[0,1)$ for a point at which $h_{j}$ attains its maximum, and then fix $J$ and define two vectors

$$
\alpha=\left(a^{J}, a^{J-1}, \ldots, a^{-J+1}, a^{-J}\right), \quad \mu=\left(m_{J}, m_{J-1}, \ldots, m_{-J+1}, m_{-J}\right) .
$$

Note that (3.2) will hold if we find a sequence $\left\{z_{\ell}\right\}$ such that $\alpha z_{\ell} \rightarrow \mu \bmod 1$ as $\ell \rightarrow$ $\infty$ (with the convention that "mod 1 " for vectors means mod 1 in each component). Kronecker's Theorem [14, Theorem 444] from number theory guarantees this by showing the trajectory $\{\alpha z \bmod 1: z \in \mathbb{R}\}$ is dense in the unit cube of $\mathbb{R}^{2 J+1}$. The transcendentality of $a$ is used here to satisfy the hypothesis of Kronecker's Theorem, namely that the entries of $\alpha$ should be linearly independent over the integers.

To prove the claims about $A_{\psi}$ in Theorem 2.1. suppose $B_{\psi}<\infty$. Then the functional $f \mapsto \sum_{j, k \in \mathbb{Z}}\left|\left\langle f, \psi_{j, k}\right\rangle\right|^{2}$ is continuous on $L^{2}$ (see [17, Lemma B.1]) so that in the definition (1.1) of $A_{\psi}$ we may replace $\{f:\|f\|=1\}$ with its dense subclass $\mathcal{C}$ :

$$
A_{\psi}=\inf _{f \in \mathcal{C}} \sum_{j, k \in \mathbb{Z}}\left|\left\langle f, \psi_{j, k}\right\rangle\right|^{2}=\inf _{f \in \mathcal{C}} \inf _{z \in \mathbb{R}} \sum_{j \in \mathbb{Z}} T_{j} f\left(a^{j} z\right),
$$

by arguing as before. Hence $A_{\psi} \geq \inf _{f \in \mathcal{C}} \sum_{j \in \mathbb{Z}} \min T_{j} f$. For the equality statement on $A_{\psi}$, apply Lemma 3.1 to $h_{j}=\left(\max T_{j} f\right)-T_{j} f$, noting that $\sum_{j \in \mathbb{Z}} \max T_{j} f$ $\leq B_{\psi}<\infty$ when $a$ is transcendental, by the equality statement for $B_{\psi}$.

\section{Proof of Corollary 2.2}

We need some further properties of the $T_{j} f$.

Lemma 4.1 ([17, Section 8]). Let $f \in \mathcal{C}$. Then $T_{j} f \in C[0,1]$ and $T_{j} f$ equals its Fourier series at every point, for each $j \in \mathbb{Z}$. Obviously $\widehat{T_{j} f}(0) \geq 0$ because $T_{j} f \geq 0$. The Fourier coefficients are given by

$$
\widehat{T_{j} f}(n)=\int_{\mathbb{R}} \overline{\hat{f}(\xi)} \hat{f}\left(\xi-n a^{j}\right) \widehat{\psi}\left(\xi a^{-j}\right) \overline{\widehat{\psi}\left(\xi a^{-j}-n\right)} d \xi,
$$

and their double sum satisfies

$$
\sum_{j \in \mathbb{Z}} \sum_{n \neq 0}\left|\widehat{T_{j} f}(n)\right|<\infty
$$

Since $T_{j} f$ equals its Fourier series at every point, $T_{j} f(z)=\sum_{n \in \mathbb{Z}} \widehat{T_{j} f}(n) e^{2 \pi i n z}$. Thus

$$
\max T_{j} f \leq \widehat{T_{j} f}(0)+\sum_{n \neq 0}\left|\widehat{T_{j} f}(n)\right|, \quad \min T_{j} f \geq \widehat{T_{j} f}(0)-\sum_{n \neq 0}\left|\widehat{T_{j} f}(n)\right| .
$$

We can substitute these bounds into Theorem 2.1. Note the resulting quantities are well defined because the double sum $\sum_{j \in \mathbb{Z}} \sum_{n \neq 0}\left|\widehat{T_{j} f}(n)\right|$ converges to a finite real number by (4.1) while $\sum_{j \in \mathbb{Z}} \widehat{T_{j} f}(0)$ converges in $[0, \infty]$ (each term being nonnegative). 
To prove the corollary we now invoke the formula for $\widehat{T_{j} f}(n)$ from Lemma 4.1 and follow the original proof of Casazza and Christensen [8, Theorem 5.1], which judiciously employs Cauchy-Schwarz on both the integral and the sum over $n$.

Remark. The Fourier expansion of the function $T_{j} f$ was developed in [17] to study completeness of orthonormal affine systems with arbitrary real dilations. For higher dimensions see [18]. The idea was then applied for quite general Gabor-affine systems in 15 .

\section{Open PROBLEMS ON FRAME CHARACTERIZATION}

Affine frames with band-limited $\psi$ were characterized by Chui and Shi [10, p. 40]. For integer $a$ and general $\psi$, Ron and Shen [19, Theorem 1.2] found a beautiful frame characterization in terms of $\ell^{2}$-norms of the dual Gramian and its inverse. Useful sufficient conditions for frames were known earlier, [11, Theorems 2.8 and 2.9] and [16, $\S 8.3]$, and later [8, Theorem 5.1]. Tight affine frames $\left(A_{\psi}=B_{\psi}\right)$ have been very successfully characterized, with the latest developments in [9, 15]. Bownik [4, §3] has extended the dual Gramian approach to rational dilations for tight frames. The nontight case seems to be open whenever $a$ is noninteger: no necessary condition is known that comes close to the sufficient ones mentioned above.

\section{REFERENCES}

1. R. Balan, P. Casazza and D. Edidin. On signal reconstruction without phase. Appl. Comput. Harmon. Anal. 20 (2006), 345-356.

2. J. J. Benedetto. Noise reduction in terms of the theory of frames. Signal and image representation in combined spaces, Wavelet Anal. Appl., 7, Academic Press, San Diego, CA, 1998, pp. 259-284. MR $1614977(99 \mathrm{e}: 94005)$

3. J. J. Benedetto and M. Fickus. Finite normalized tight frames. Frames. Adv. Comput. Math. 18 (2003), no. 2-4, 357-385. MR.1968126 (2004c:42059)

4. M. Bownik. Quasi-affine systems and the Calderón condition. Harmonic analysis at Mount Holyoke (South Hadley, MA, 2001), Contemp. Math., 320, Amer. Math. Soc., Providence, RI, 2003, pp. 29-43. MR1979930 (2004h:42039)

5. P. G. Casazza. The art of frame theory. Taiwanese J. Math. 4 (2000), no. 2, 129-201. MR:1757401 (2001f:42046)

6. P. G. Casazza. Custom building finite frames. Wavelets, frames and operator theory, Contemp. Math., 345, Amer. Math. Soc., Providence, RI, 2004, pp. 61-86. MR2066822 (2005f:42078)

7. P. G. Casazza and O. Christensen. Weyl-Heisenberg frames for subspaces of $L^{2}(\mathbb{R})$. Proc. Amer. Math. Soc. 129 (2001), no. 1, 145-154. MR1784021 (2001h:42046)

8. O. Christensen. Frames, Riesz bases, and discrete Gabor/wavelet expansions. Bull. Amer. Math. Soc. (N.S.) 38 (2001), no. 3, 273-291. MR1824891 (2002c:42040)

9. C. K. Chui, W. Czaja, M. Maggioni and G. Weiss. Characterization of general tight wavelet frames with matrix dilations and tightness preserving oversampling. J. Fourier Anal. Appl. 8 (2002), no. 2, 173-200. MR1891728 (2003a:42038)

10. C. K. Chui and X.-L. Shi. Bessel sequences and affine frames. Appl. Comput. Harmon. Anal. 1 (1993), no. 1, 29-49. MR.1256525 (95b:42028)

11. I. Daubechies. The wavelet transform, time-frequency localization and signal analysis. IEEE Trans. Inform. Theory 36 (1990), no. 5, 961-1005. MR1066587 (91e:42038)

12. R. J. Duffin and A. C. Schaeffer. A class of nonharmonic Fourier series. Trans. Amer. Math. Soc. 72 (1952), 341-366. MR0047179(13:839a)

13. K. Gröchenig. Foundations of Time-Frequency Analysis. Birkhäuser, Boston, 2001. MR:1843717 (2002h:42001)

14. G. H. Hardy and E. M. Wright. An Introduction to the Theory of Numbers. Third edition. Oxford University Press, London, 1954. MR0067125 (16:673c) 
15. E. Hernández, D. Labate and G. Weiss. A unified characterization of reproducing systems generated by a finite family. II. J. Geom. Anal. 12 (2002), no. 4, 615-662. MR1916862 (2003j:42036)

16. E. Hernández and G. Weiss. A First Course on Wavelets. CRC Press, Boca Raton, Florida, 1996. MR 1408902 (97i:42015)

17. R. S. Laugesen. Completeness of orthonormal wavelet systems for arbitrary real dilations. Appl. Comput. Harmon. Anal. 11 (2001), no. 3, 455-473. MR.1866351 (2002h:42073)

18. R. S. Laugesen. Translational averaging for completeness, characterization and oversampling of wavelets. Collect. Math. 53 (2002), no. 3, 211-249. MR.1940326 (2003i:42053)

19. A. Ron and Z. Shen. Affine systems in $L_{2}\left(\mathbb{R}^{d}\right)$ : the analysis of the analysis operator. J. Funct. Anal. 148 (1997), no. 2, 408-447. MR.1469348 (99g:42043)

Department of Mathematics, University of Illinois, Urbana, Illinois 61801

E-mail address: Laugesen@uiuc.edu 